\title{
CONTROL SYSTEM OF VEHICLE FOR SMART FACTORY MODEL WiTH PRINCIPLES OF INDUSTRY 4.0
}

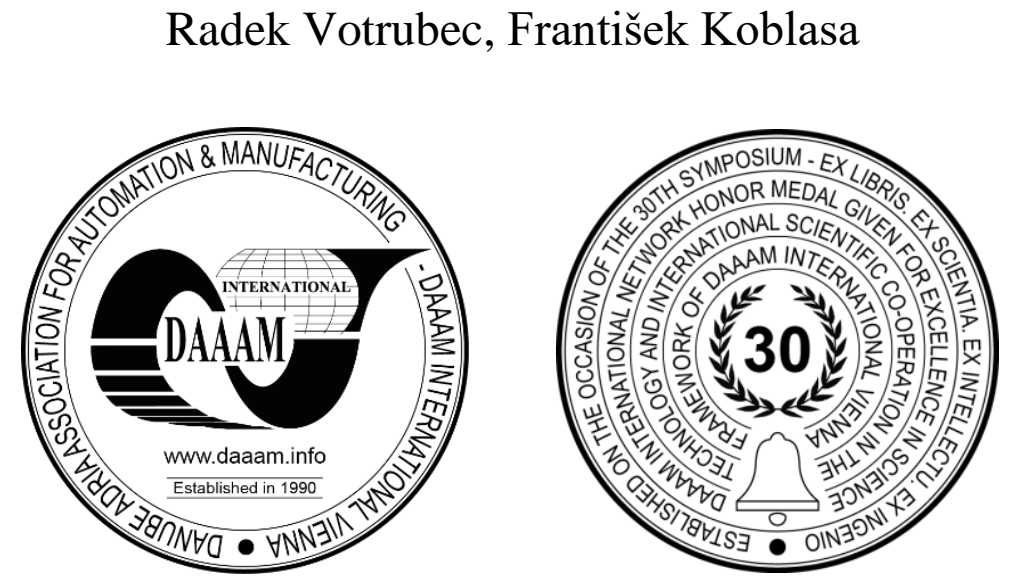

This Publication has to be referred as: Votrubec, R[adek] \& Koblasa, F[rantisek] (2019). Control System of Vehicle for Smart Factory Model with Principles of Industry 4.0, Proceedings of the 30th DAAAM International Symposium, pp.0261-0267, B. Katalinic (Ed.), Published by DAAAM International, ISBN 978-3-902734-22-8, ISSN 1726-9679, Vienna, Austria

DOI: $10.2507 / 30$ th.daaam.proceedings.034

\begin{abstract}
This paper aims at making an accurate control system for an educational model of smart factory vehicle. The smart factory vehicle is aimed at communicating with the other members of the smart factory using Wi-Fi communication technique. This targets to eliminate any human interference. Therefore it ensures there is no deviation from target which may arise due to human negligence, fatigue or performance efficiency of an individual. The purpose of the vehicle is to carry small subjects from one place to another as per requirements of the factory. The vehicle will be equipped with various sensors aiming at automated guiding of the vehicle.
\end{abstract}

Keywords: Smart factory; Arduino; Industry 4.0; Wifi communication; automation.

\section{Introduction}

At our department we are currently creating a model of a production system suitable for teaching the modern principles of Industry 4.0. [1],[2]. In 2012 the German government made "Industry 4.0" one of the 10 future projects of their hightech strategy [3]. The evolution of Industry 4.0 in Europe and East Asia is described in [4]. Described strategies could be valuable for each company [5],[6]. The most important requirement of the model is the possibility to show the difference between the Smart Things concept and central control. Individual components of the emerging model are not centrally controlled, but each element has its own control system and communicates with the other elements of the factory.

The smart factory vehicle has to perform several functions in the smart factory for the work flow to happen. The smart factory vehicle has to travel on the track provided in the smart factory. The track is split into two loops, inner loop and outer loop. The decision of inner loop or outer loop must be decided on Wi-Fi based on the work process. If the process of goods collection is finished, the vehicle has to exit the inner loop and join the outer loop and stop at the point where the goods are delivered. There are two arduinos involved in this process. One arduino is on board the vehicle to receive information on the work flow. The other arduino is the transmitter of information whether the work is still pending or the vehicle can reach the delivery point. The vehicle itself must be constructed sturdy with all the connections intact. The components have to be properly fastened and the wiring must be done according to the module under consideration. Loose contacts in wiring must be taken extra care as they may result in error in values. There are two Wi-Fi modules which are 
used for the communication process. One Wi-Fi module is on the vehicle which is the receiver. The other Wi-Fi module is on the vehicle which acts as the transmitter.

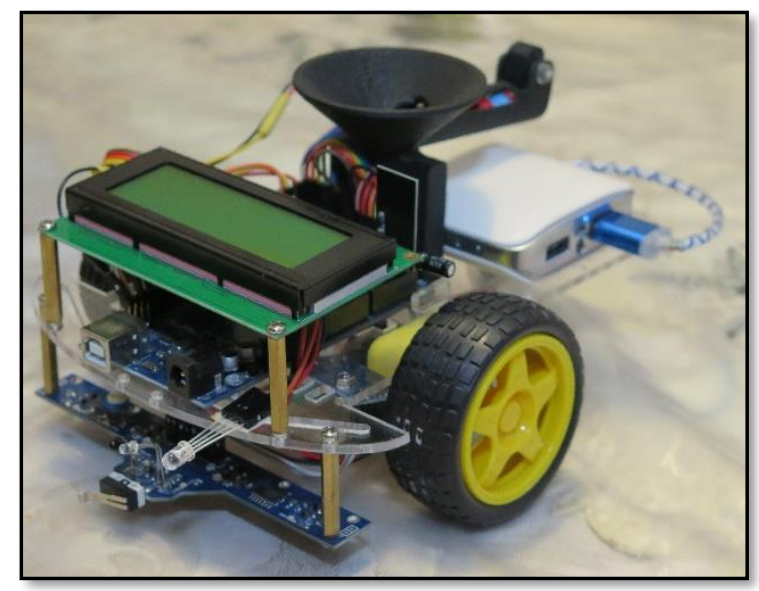

Fig. 1. Smart Factory Vehicle

There are various components or modules used in the vehicle. The components such as tracking sensor, colour sensor, single IR Sensor should be mounted on the vehicle in a stable manner. The output values change according to the height at which they are placed from the ground level. There is an onboard power bank which supplies the input voltage required for the functioning of the modules. A breadboard is used to enable wiring of all modules undisturbed.

\section{Model of Smart Factory}

The scheme of the smart factory is explained diagrammatically in the picture shown in figure 2 . The smart factory is based on Industry 4.0 concept. The main function of this smart factory is transportation of goods from one place to another. There are six provisions in the smart factory where goods are delivered from the provision to the vehicle. There are five vehicles where they go around the track collecting goods from the provision.

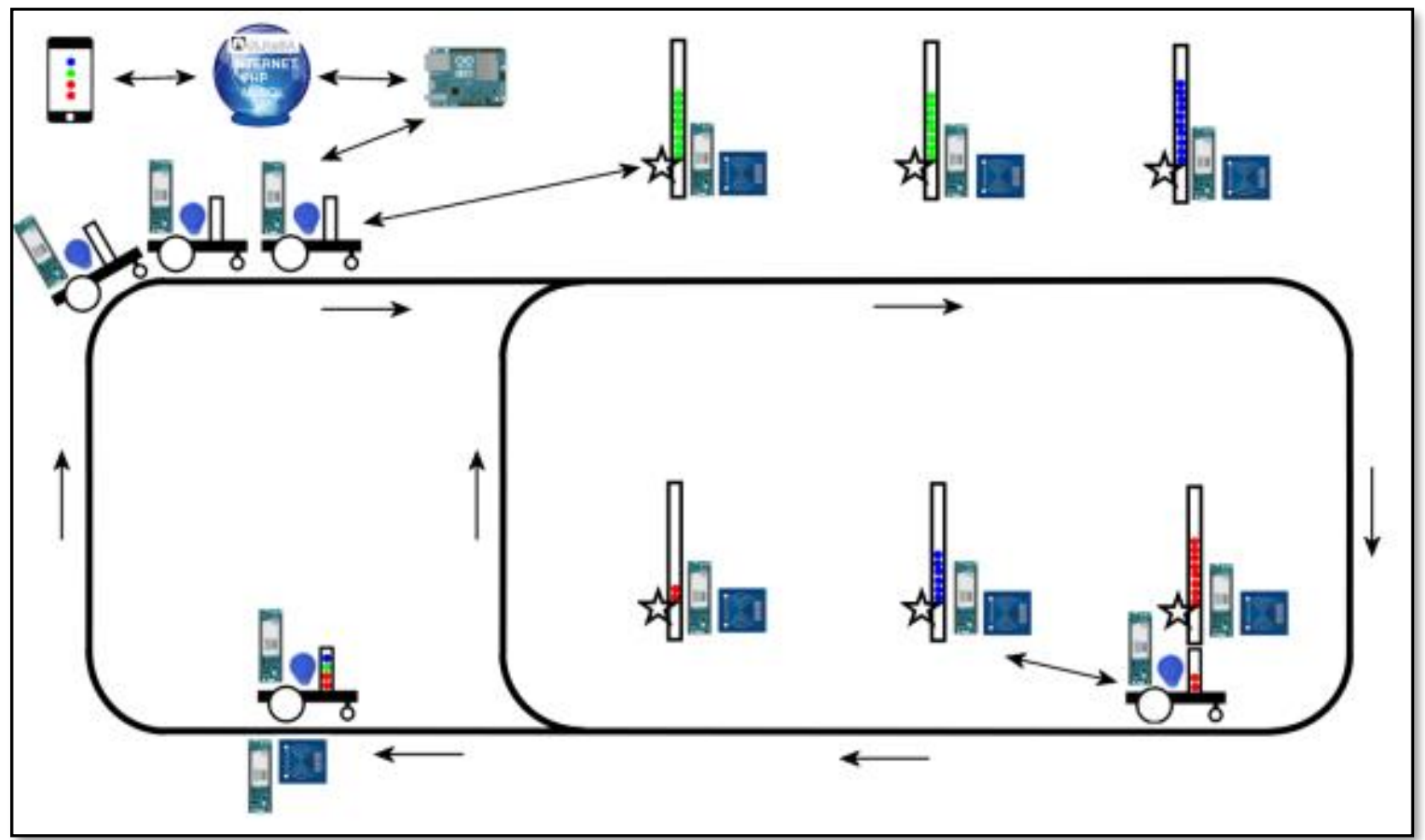

Fig. 2. Scheme of Smart Factory 
Each vehicle receives the information on the combination of goods to be collected from the provisions and starts the line following. This information is received by the vehicle through a Wi-Fi module on board the vehicle. The vehicle also receives the information on the next available station. The process starts by the vehicle entering the inner loop. After receiving the combination of goods, it starts to travel in the loop and continues to travel on the inner loop until all the goods are on board. Once it is finished, the vehicle leaves the loop and travels to the desired position where the goods have to be delivered. A photograph of the model of our smart factory is in figure 3.

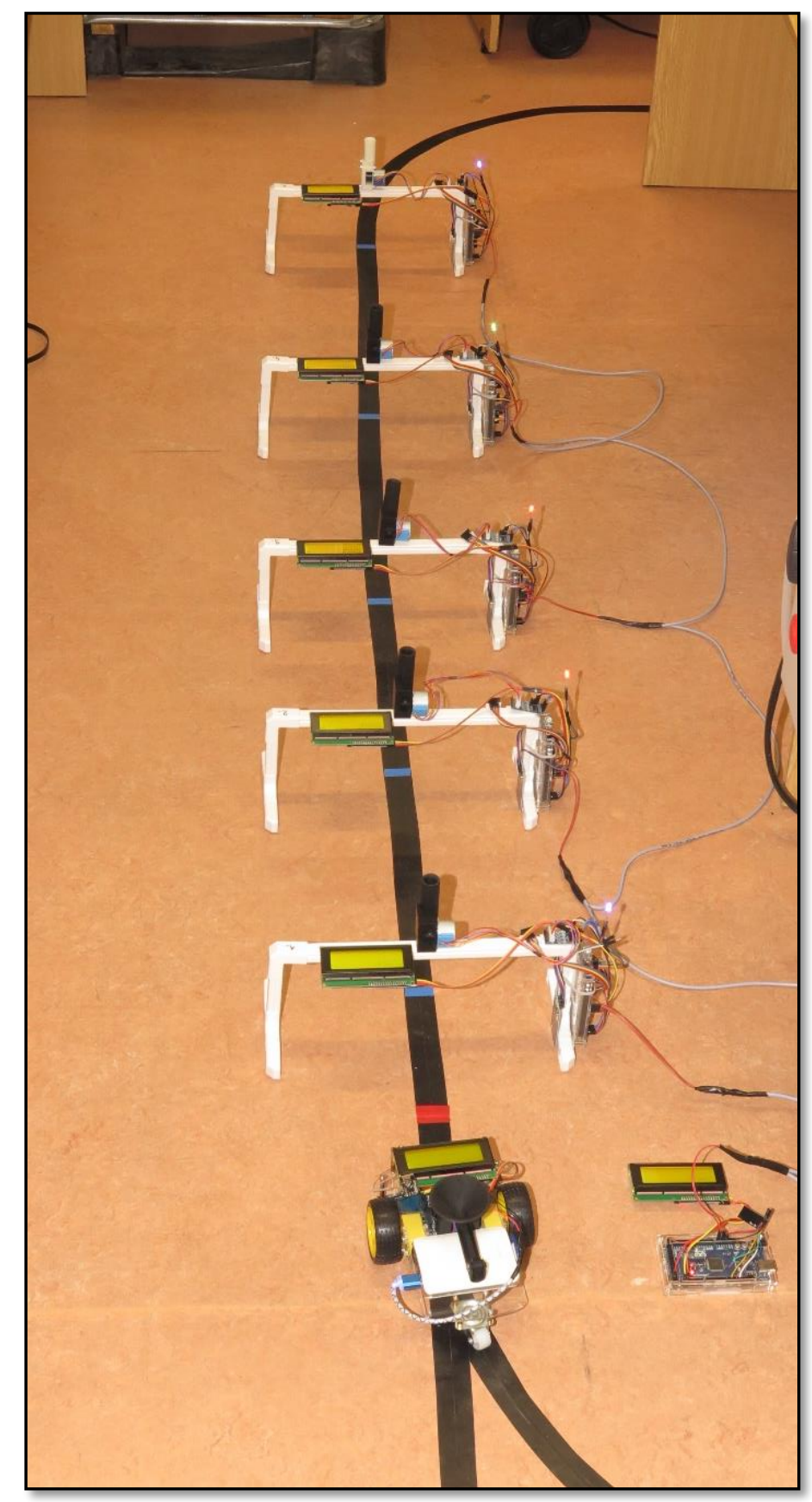

Fig. 3. The model of smart factory

\section{Line Folowing}

This vehicle is capable of following a line without the help of any external source. It has 5-infrared sensors on the bottom for detection of black tracking tape. As we know that black colour is capable of absorbing the radiation and white colour or a bright colour reflects the radiation back. Here we use 5 pairs of IR Tx and Rx .The robot uses these IR sensors to sense the line and the arrangement is made such that the sensors face the ground. 
The output from the sensors is an analog signal which depends on the amount of light reflected back and this analog signal is given to the comparator to produce binary signal. Controller of black-line tracker use three sensors. The center sensor detects whether the vehicle is on the line and the two side sensors detect the line edge. We can notice that the function of line following has a dependency on the thickness of the line being used for track navigation and then it depends on the distance of the sensor before wheel axis. As the thickness of the line decreases, the deviation is less until a certain point beyond which it again increases. The sensor spacing may be greater, equal to or less than the line width. If the sensors are closer, the vehicle is better fixed to the line, figure $4 \mathrm{a}$. If the sensors are further away, the vehicle runs in curves, figure $4 \mathrm{~b}$.

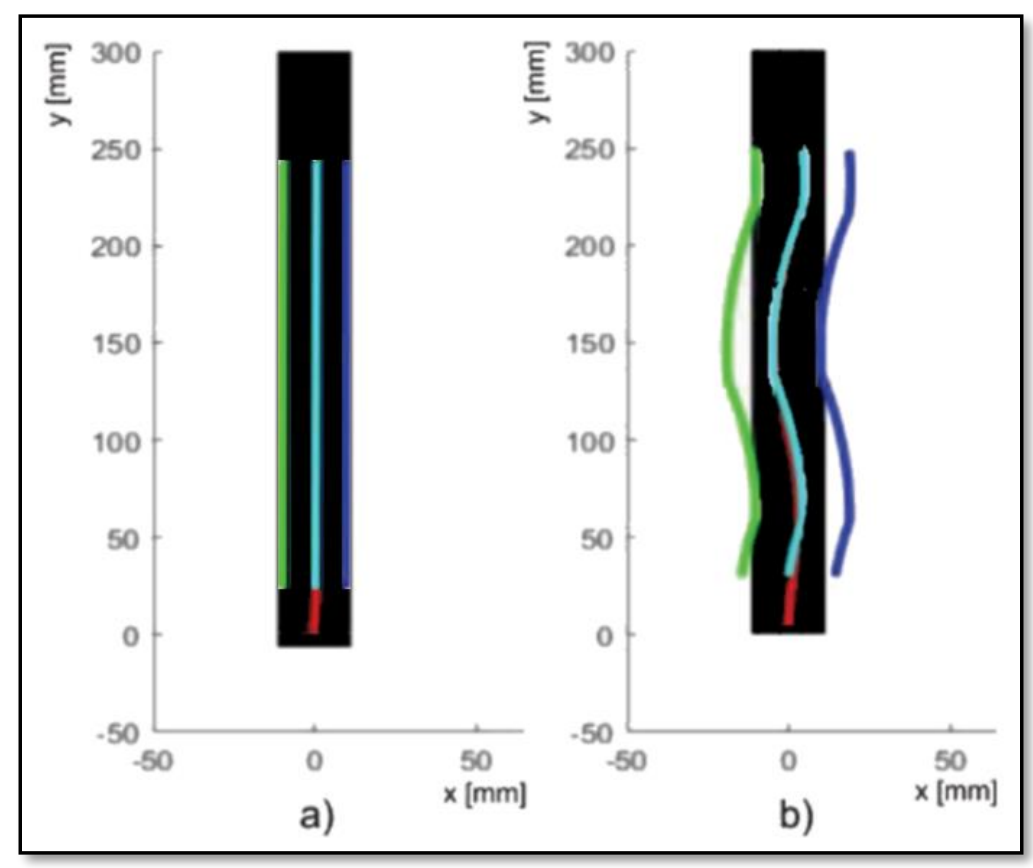

Fig. 4. Dependency of movement on distance between sensors

If the sensors are further in front of the vehicle, the vehicle has a smaller oscillation $\mathrm{m}$, figure 5a, than in the case if sensors are near, figure 5b. According to source [8] this is currently being used for higher vehicle speed because they drive more directly. A detailed analysis of the influence of the sensor position is at [7].

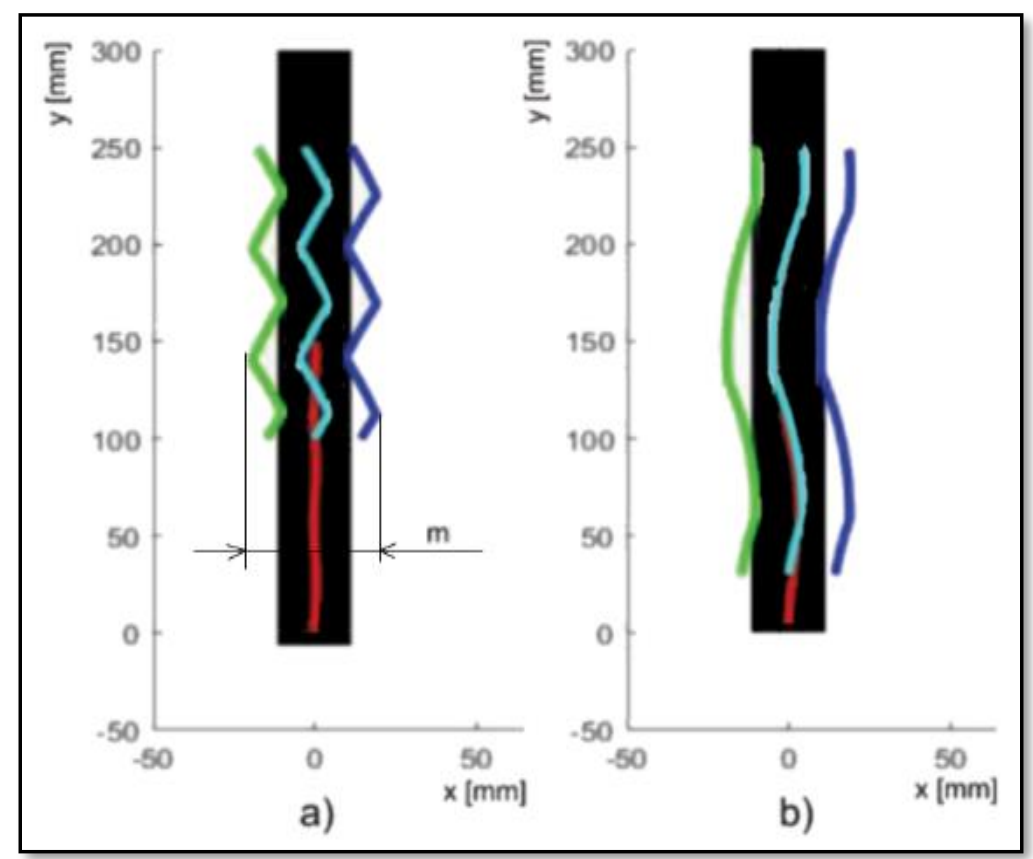

Fig. 5. Dependency of movement on placement of sensor before wheel axis 


\section{Colour detection}

This module is very interesting and can be used to detect colours when in close proximity. The module consists of four white colour LED's which are used to illuminate the subject under study and has a large number of photodiodes that filters colours.

The colour sensor contains RGB (Red Green Blue) arrays. Each of these arrays contain three sensors, one is for sensing RED light intensity, one is for sensing GREEN light intensity and the last one for sensing BLUE light intensity. The transmitter $\mathrm{Wi}-\mathrm{Fi}$ module communicates a colour pattern before the start of the process, the receiver (vehicle) receives this pattern and traverses the path accordingly.

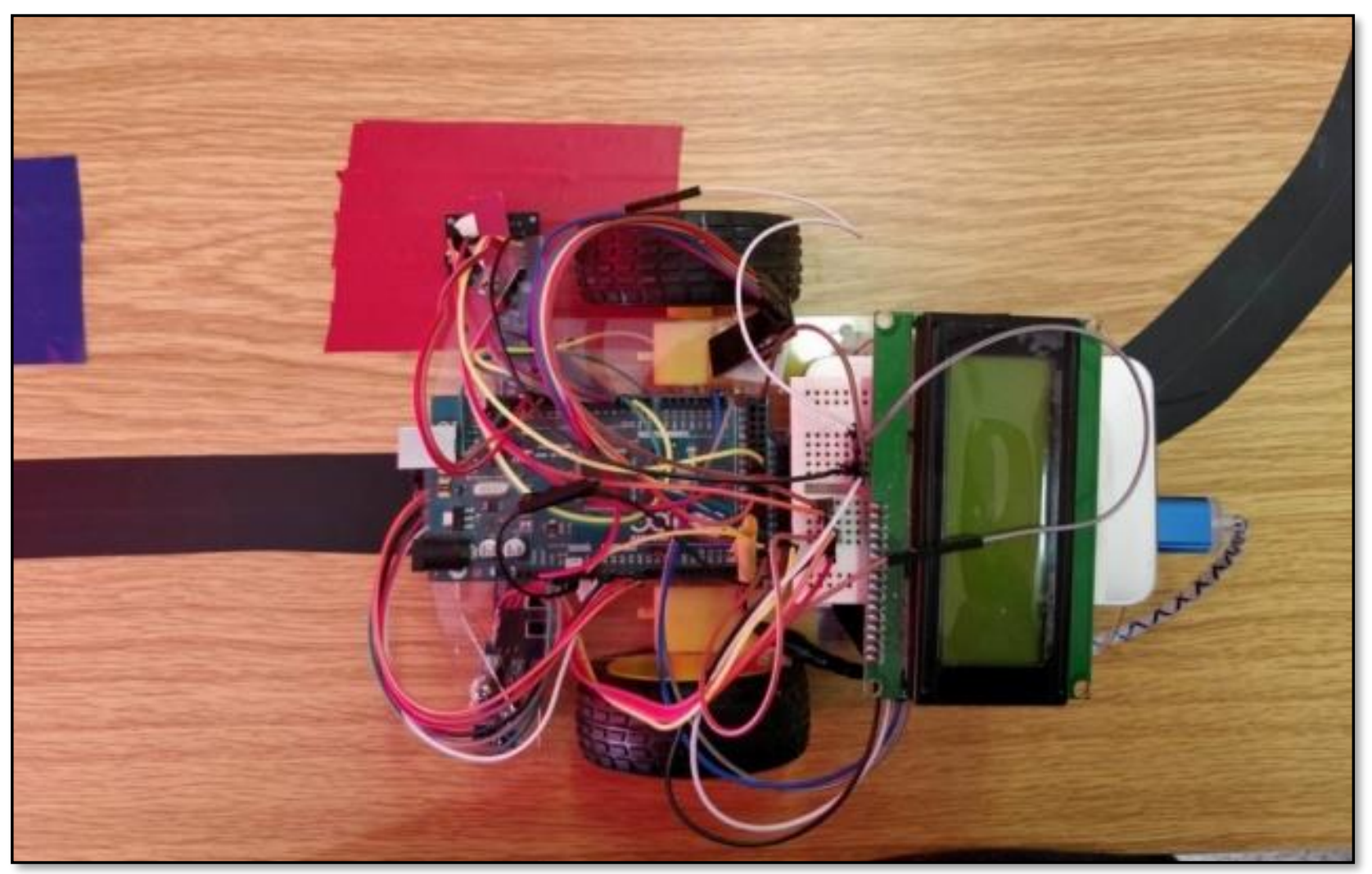

Fig. 6. Colour detection

\section{Wifi communication}

Several Wi-Fi modules have been employed in this project. At the moment one Wi-Fi module works as a transmitter while the other acts as a receiver. The receiver is connected to the arduino on the vehicle whereas the transmitter is connected to another arduino that is stationary and used by the user to send commands to the vehicle. At the beginning of the process, the transmitter $\mathrm{Wi}-\mathrm{Fi}$ module will send the colour pattern to the vehicle.

The vehicle captures this pattern and traverses the loops according to this pattern. While the vehicle is traversing the loops, at the junction of demerging curves, the vehicle needs to decide on which direction to take.

For instance, if the customer offers a pattern $<$ Red, Blue, Red $>$, the vehicle follows the black line while constantly searching for the colour RED, once the colour sensor senses the RED colour, the vehicle is programmed to stop at that location and asks the delivery station fixed at that location to transfer the goods to the vehicle. The vehicle then traverses the line searching for BLUE colour and then finally searches for RED again and follows the same procedure. The vehicle chooses the outer loop at the demerging point after collecting the pattern. Special communication protocol was developed. The function is described via automaton graphs, figure 7. The communication code consists of several commands and parameters. There are three layers of wifi communication. First it is physical hardware layer. Second layer consists of basic functions to communicate, send, receive and confirm the messages. Finally there is third layer which is main program, sequential automaton. 


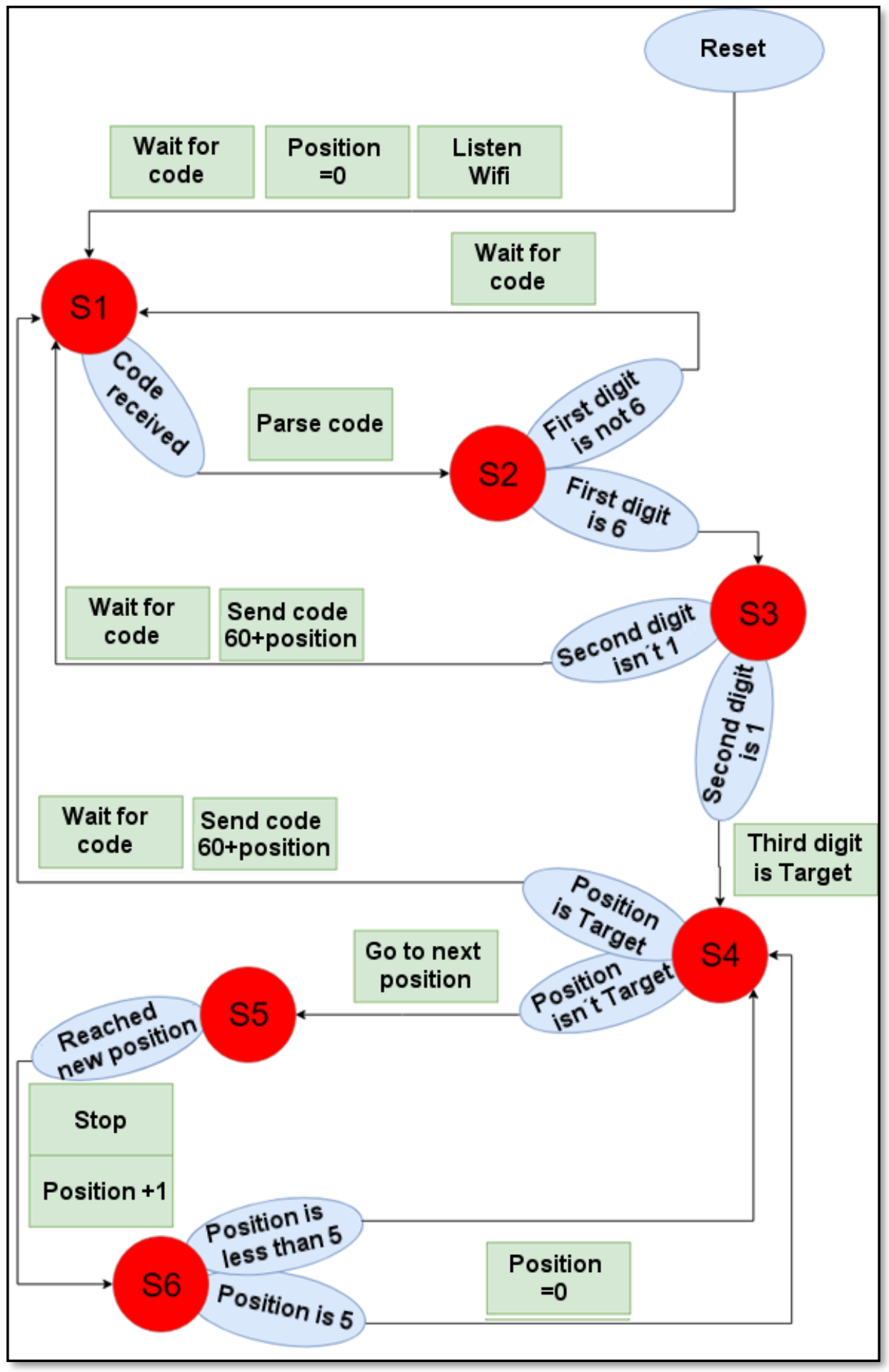

Fig. 7. An example of automaton graph of the vehicle

\section{Conclusion}

This paper focused on two issues. The first issue is the optimization of vehicle driving based on black line tracking. An analysis of the influence of sensor position on driving properties was performed. We needed to increase the accuracy of the ride in the transit stations, where it is necessary to handle the bead and place it correctly on the vehicle. At the same moment it is necessary to register the RFID chip in the site. The range of the RFID reader is 10-15mm, so the vehicle must drive straight through the station without curves. This was achieved by appropriate sensor spacing with respect to line width and correct placement in front of the vehicle. The second part focuses on the structure of educational model of smart factory with manufacturing process of bracelet assembly using multiple cartridges and carts. The biggest task was to design and implement a protocol of wifi communication between individual elements of the factory. 
Function libraries were created and a way of communication was designed. The relationships between the components were described with graphs of automata. The model of smart factory is used to research and teaching of manufacturing processes. Now we are developing the strategies using artificial intelligence. This solution is also suitable for other applications. It is going to be used for the other manufacturing system on our department. Because arduino is used as the control elements, the system is suitable for learning with the possibility of extension in the framework of working with students. Principles would remain for industrial use, but arduino would be replaced by industrial PLCs.

\section{Acknowledgments}

This publication was written at the Technical University of Liberec as part of the project "(21278) - Optimization of manufacturing systems, 3D technologies and automation“" with the support of the Specific University Research Grant, as provided by the Ministry of Education, Youth and Sports of the Czech Republic in the year 2019

\section{References}

[1] Bartodziej, Ch. J., (2016), The concept industry 4.0. New York, NY: Springer Berlin Heidelberg, 2016. ISBN 9783-658-165017.

[2] Thanes, L., Schaefer, D., (2017) Cybersecurity for Industry 4.0: Analysis for Desing and Manufacturing. Switzerland: Springer International Publishing, 2017. ISBN 978-3-319-50659-3.

[3] Bundesministerium fuer Bildung and Forschung, (2014). Die neue Hightech-Strategie. Innovationen fuer Deutschland, Berlin

[4] Takakuwa, S.; Veza, I. \& Celar, S. (2018). Industry 4.0 in Europe and East Asia, Proceedings of the 29th DAAAM International Symposium, pp.0061-0069, B. Katalinic (Ed.), Published by DAAAM International, ISBN 978-3902734-20-4, ISSN 1726-9679, Vienna, Austria DOI: 10.2507/29th.daaam.proceedings.009

[5] Palcic, I.; Ojstersek, R. \& Buchmeister, B. (2018). Creating Value in Manufacturing Companies, Proceedings of the 29th DAAAM International Symposium, pp.0070-0075, B. Katalinic (Ed.), Published by DAAAM International, ISBN 978-3-902734-20-4, ISSN 1726-9679, Vienna, Austria DOI: 10.2507/29th.daaam.proceedings.010

[6] Medojevic, M.; Diaz Villar, P.; Cosic, I.; Rikalovic A.; Sremcev, N. \& Lazarevic, M. (2018). Energy Management in Industry 4.0 Ecosystem: a Review on Possibilities and Concerns, Proceedings of the 29th DAAAM International Symposium, pp.0674-0680, B. Katalinic (Ed.), Published by DAAAM International, ISBN 978-3-902734-20-4, ISSN 1726-9679, Vienna, Austria DOI: 10.2507/29th.daaam.proceedings.097

[7] Manlig, F. (2019), Optimalization of processes of educational model of smart factory with principles of Industry 4.0. Master thesis, Technical university of Liberec, Czech Republic, 2019

[8] Ullrich, G. (2014). Automated guided vehicle systems, New York, Springer, ISBN9783662448137 